\title{
THE ROLE OF LIGHT-INDUCED FLUORESCENCE IN THE TREATMENT OF SMOOTH SURFACE CARIOUS LESIONS WITH ICON INFILTRATION AND THE RESULTS AFTER 1 YEAR
}

\author{
R. Kabaktchieva, N. Gateva and K. Peycheva \\ Departmen Conservative Dentistry, Faculty of Dental Medicine, Medical University - Sofia
}

Summary. Caries infiltration is a novel technique that brings out immediate esthetic improvement in the opacity of the white spot lesions. Light-induced fluorescence method is a modern caries diagnostic method. In this study SoproLife camera (Acteon, France) was applied for diagnosing and follow-up of the results. The aims of this in vivo study are to test the role of light-induced fluorescence method (SoploLife camera) in the diagnosis of non-cavitated smooth surfaces carious lesions (ICDAS codes 1 and 2) of primary and permanent teeth and in the follow-up period immediately after application, 6 months and 1 year after applying ICON material (DMG). Teeth: $\mathrm{n}=90$; primary teeth: 6 kids; $\mathrm{n}=40$ teeth; permanent teeth: 6 patients; $\mathrm{n}=50$ teeth. Visual examination by ICDAS without probe, dry for $10 \mathrm{~s}$ with 3-in-1 syringe using lightening; SoproLife camera $(450 \mathrm{~nm})$, digital photos. LIF method applied with SoproLife camera (Diagnostic mode with day light and blue light) is more accurate than visual examination only when applied for single tooth diagnose. Moreover, LIF method for single tooth is more accurate in following up the effect of non-operative treatment of smooth surfaces lesions than using digital images. ICON is a material that stops the progression of non-cavitated smooth surfaces carious lesions in both primary and permanent teeth and make the aesthetic result better up to 1 year following the procedure.

Key words: caries infiltration, white spot, microinvasive, noncavitated lesion

\section{INTRODUCTION}

We cycle of de-remineralization is an ongoing phenomenon and takes place in oral cavity with intake of various foods or drinks. But if the oral protective factors, e.g., salivary buffers, fluorides, etc., are unable to keep the balance in favor of remineralization, there occurs a net loss of mineral 
leading to incipient caries lesion [1, 2, 16]. Causes of white spot lesion may include heavy plaque accumulation, inadequate oral home care routines and a high sugar or acid content diet, removal of orthodontic bands and brackets. They are frequently called as enamel scars (6). White spot lesions are early signs of demineralization under intact enamel, which may or may not lead to the development of caries. Smooth surfaces are classical areas of white spot lesions lose their translucency.

Dentists have always found it difficult to deal with the white spot lesions in esthetic zones. These early lesions are amenable to remineralization or arrest but if the demineralization process is not stopped, the intact enamel surface eventually collapses and cavitates [20]. The treatment of ICDAS II codes 1 and 2 /white spots/ is remineralization. There are creams, pastes and topical remineralization treatments such as fluoride therapy, casein-phosphopeptide-amorphous calcium phosphate pastes, invasive approaches such as microabrasion, conventional bonding [5]. A new minimally invasive technique for treating white spot lesions is a product of "DMG," called "Icon" which uses low-viscosity light-curing resins for caries infiltration. Not like sealants and bonds that cover the surface of the lesion, Icon rapidly penetrates into the pores of the body of the lesion and closes them. Thus, paths for bacterial metabolism are being blocked, and the resin completely fills the pores within the tooth, replacing the lost tooth structure and stopping caries progression [3, $6,20]$. The treatment is done in one simple visit with no drilling or anesthesia needed while preserving healthy tooth structure.

The aims of this in vivo study are to test the role of light-induced fluorescence method (SoploLife camera) in the diagnosis of non-cavitated smooth surfaces carious lesions (ICDAS codes 1 and 2 ) of primary and permanent teeth and in the followup period immediately after application, 6 months and 1 year after applying ICON material (DMG).

\section{MATERIAL AND METHODS}

Teeth: Smooth surfaces carious lesions, $n=90$. Primary teeth: 6 kids; $n=40$ teeth, Permanent teeth: 6 patients; $n=50$ teeth.

Diagnostics: Oral prophylaxis with brush and paste without fluoride is done before examination. Visual examination by ICDAS without probe, dry for $10 \mathrm{sec}$. SoproLife camera (Acteon, France) - light-induced fluorescence method (LIF) for early detection of caries using diagnostic mode - day light and blue light $(450 \mathrm{~nm})$. Color scale of the camera with blue light: blue or green = sound enamel, white color = demineralization; red or black color $=$ carious lesion. Daylight serves as an intraoral camera with magnification $\times 30$ to $\times 100$ times. Photos by digital camera are taken of professional dental photographer.

Application Protocol: 1. Clean the teeth, place retractor and gingival barrier. 2. Apply Icon etch (Chydrochloric acid 16\%) for 2 min. 3. Rinse with water for $30 \mathrm{~s}$. Dry with air. 4. Apply Icon Dry (ethanol) for $30 \mathrm{~s}$. 5. Apply Icon-Infiltrant for 3 min. 6 . Light cure for $40 \mathrm{~s}$. 7. Apply Icon-Infiltrant for $1 \mathrm{~min}$. 8. Light cure for $40 \mathrm{~s}$. 
Follow up - photos by digital camera and two photos of each lesion by SoproLife camera with daylight and blue light in "diagnostic" mode were taken immediately after application of Icon material, $1^{\text {st }}$ month, $3^{\text {rd }}$ month and $6^{\text {th }}$ month after. Results were also controlled by visual examination with and without drying and by digital camera photos.
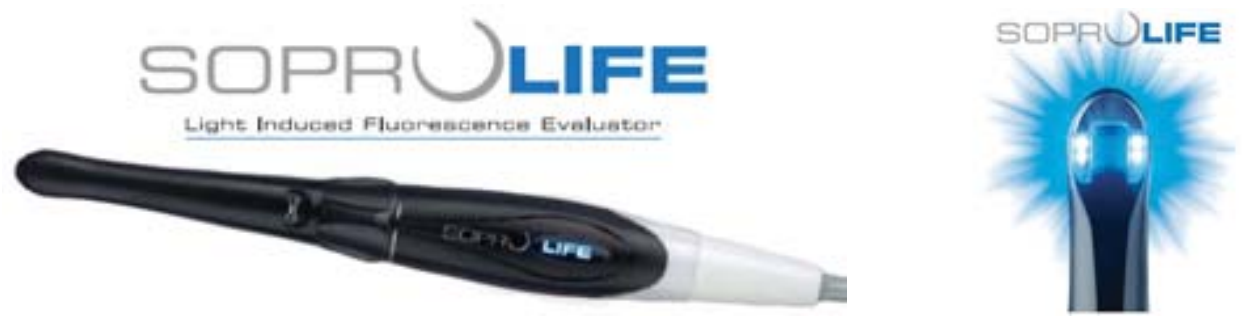

Fig. 1. Image of SoproLife camera

\section{RESULTS}

\section{Results in Primary Teeth, $\mathrm{n}=\mathbf{4 0}$}

Visual and digital control. In five teeth there was no difference between the beginning and the follow-ups even after 1 year. But the caries was hold at the same level which is some success; in 2 teeth the result was worse compared to the image in the beginning; in 33 teeth the aesthetic result was better and remained good for 1 year.

\section{Results in Permanent Teeth, $\mathbf{n}=\mathbf{5 0}$}

Visual and digital control. In 11 teeth there was no difference between the beginning and the follow-ups even after 1 year. But the caries was hold at the same level, which is some success; in 39 teeth the aesthetic result was better and remained good for 1 year.

3. Results of light-induced fluorescence method (SoproLife camera) diagnostic mode with blue light

In 16 primary and 19 permanent teeth, that were visually diagnosed successfully, the camera still detects demineralization - white fluorescence color, but the spot was smaller and with low intensity of the color after Icon. The explanation could be: in deeper demineralization Icon could not infiltrate the deepest part of the body of the lesion and the camera again detects demineralization because the light goes to these deepest empty pores. Thus the lesion progression could be detected very precisely. When the demineralization is slight Icon fills all the pores in the body of the lesion and the camera detects sound tissues - green color. 
SoproLife camera - diagnostic mode with day light and blue light (450 nm)

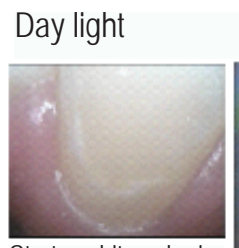

Start - white color is demineralization

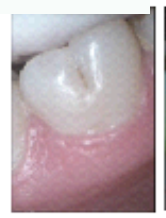

Start - white and black color is demineralization
Day light Blue light

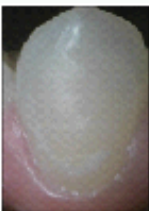

After application of ICON

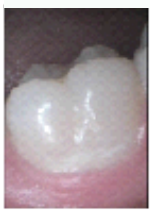

After application of ICON
Day light Blue light

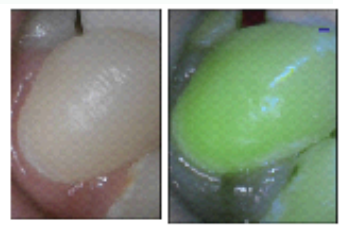

After 6 months

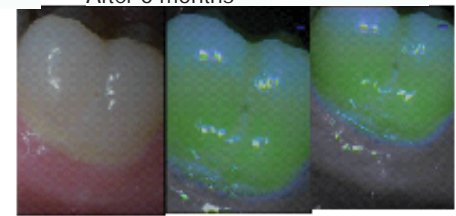

After 1 year

Fig. 2. Pictures with SoproLife: 1 - diagnostic photo, 2 - immediately after application, 3 - result after 3 months
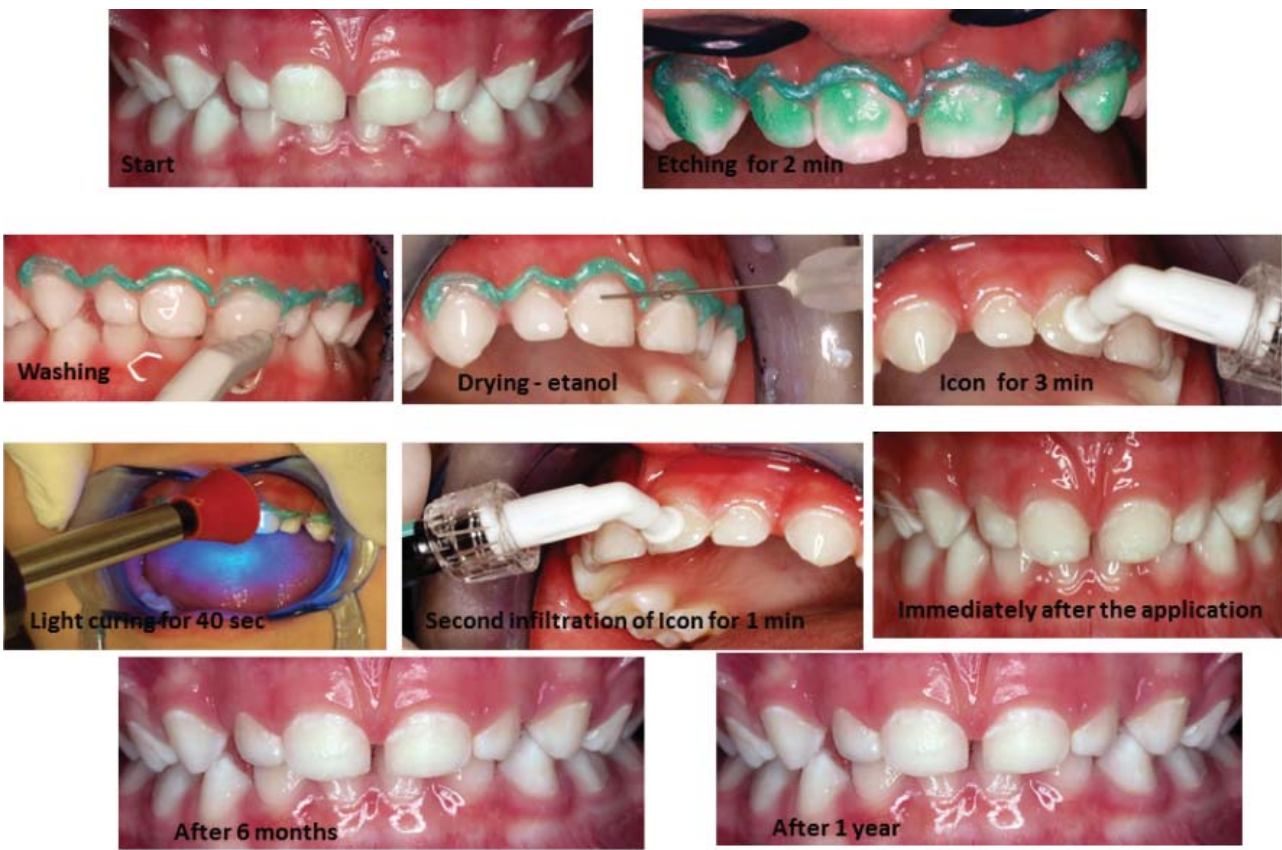

Fig. 3. Application protocol ICON in Deciduous Teeth - Smooth Surfaces Lesions 

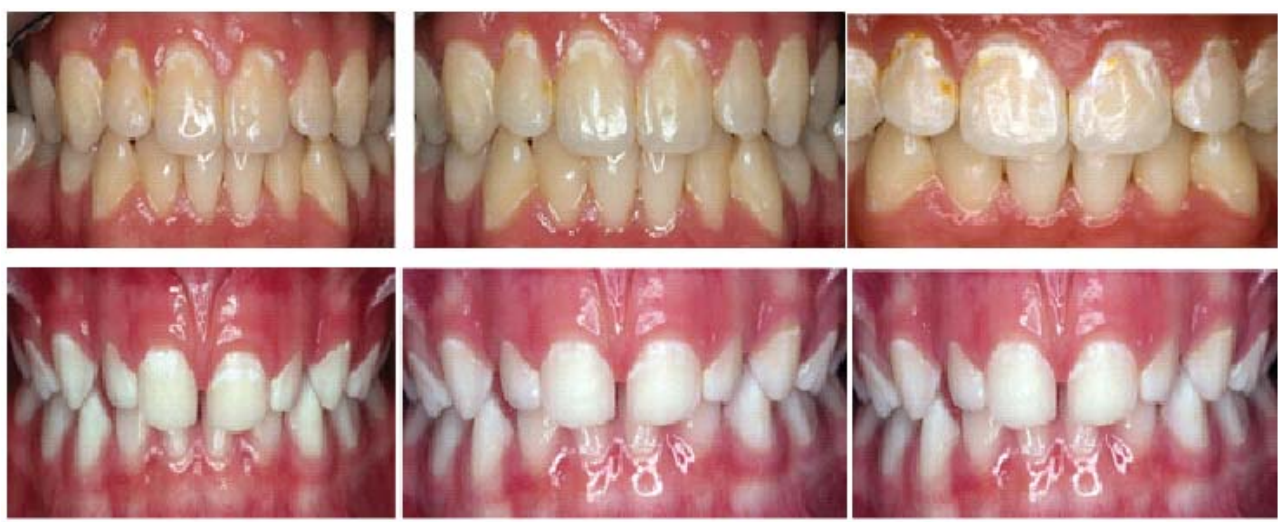

Fig. 4. Photos by camera of permanent upper frontal teeth: 1-diagnostic photo,

2- immediately after application, 3- result after 3 months

\section{DISCUSSION}

Air and water entrapments caused by the caries process have a lower refraction index than healthy hard tissue. This leads to unesthetic discolorations or so-called white spots. The infiltrant has a similar refraction index as sound enamel and can therefore equalize the difference in light refraction and approximate the appearance of the infiltrated lesion to that of the surrounding healthy enamel $[8,9]$. Application of hydrochloric acid as an etchant has been demonstrated to be superior to $37 \%$ phosphoric acid gel in removing the surface layer of natural enamel lesions when applied for 120 seconds. Moreover, in contrast to enamel microabrasion, only $30-40 \mathrm{~mm}$ are eroded with this technique, making this technique to be truly microinvasive [11].

Lueckel and Paris [13] emphasized that the original aim of caries infiltration is to arrest the lesion progression by occluding the microporosities that provide diffusion pathways for acids and dissolved minerals. The principle of masking enamel lesions by resin infiltration is based on changes in light scattering within the lesions [12]. Further Lueckel and Paris demonstrated the inhibition of caries progression both in vitro and in vivo lesions, after the application of ICON infiltrants [16]. Immediate improvement in the esthetic appearance is observed by diminishing the opacity $[4,8,18,20]$.

Following-up the results there was no progression of white spot lesion and there was an improvement in the esthetics on clinical examination. This technique may be considered as a microinvasive treatment of smooth-surface white spot lesions and also one that allows for the recovery of natural tooth appearance [10,13, $15,19]$.

Combining this ultraconservative restorative approach (which is considered microinvasive) with a substantial caries remineralization program may provide thera- 
peutic benefits and significantly reduce both long-term restorative needs and costs, thus complementing the concept of minimal intervention dentistry [7, 12].

Light-induced fluorescence method is a modern and new diagnostic method. Sopro Life camera has the abilty to diagnose caries lesions and point to the level of excavation during cavity preparation. The camera is easy to apply and emits blue light at $450 \mathrm{~nm}$. Its capability has been proven to diagnose initial caries lesions with smooth and occlusal surfaces [14, 17].

\section{CONCLUSION}

LIF method applied with SoproLife camera (Diagnostic mode with day light and blue light) is more accurate than visual examination only when applied for single tooth diagnose. Moreover, LIF method for single tooth is more accurate in following up the effect of non-operative treatment of smooth surfaces lesions than using digital images. ICON is a material that stops the progression of non-cavitated smooth surfaces carious lesions in both primary and permanent teeth and make the aesthetic result better up to 1 year following the procedure. ICON technique might be an alternative to microabrasion and restorative treatment in treating white spot lesions of esthetically relevant teeth.

\section{REFERENCES}

1. Allis on, J.et al. The disease dental caries: Risk assessment/strategies of disease control.http:// www1.umn.edu/dental/courses/dent_6806fall04/paper 1/paper1.pdf

2. Burne, R. A. et R. E. Marquis RE. Alkali production by oral bacteria and protection against dental caries. - FEMS Microbiol. Lett., 193, 2000, 1-6.

3. Kug el, G., P. Arsenaul et A. Papas A. Treatment modalities for caries management, including a new resin infiltration system. - Compendium, 30, 2009, 1-10.

4. Kugel G, Arsenaul P, Papas A. Treatment modalities for caries management, including a new resin infiltration system. Compendium.2009;30:1-10.

5. G I a z e r, H. S. Treating white spots: New caries infiltration technique. 2009.http://www.dentistrytoday.com/restorative/minimallyinvasivedentistry/1492.

6. Ha m mad, S. M. et al. Effect of resin infiltration on white spot lesions after debonding orthodontic brackets. - Am. J. Dent., 25, 2012, 3-8.

7. Kielbas sa, A. M., J. Muller et C. R. Gernhardt. Closing the gap between oral hygiene and minimally invasive dentistry: a review on the resin infiltration technique of incipient (proximal) enamel lesions. - Quintessence Int., 40, 2009, № 8, 663-681.

8. Meyer-LuekeI, H. et S. Paris. Progression of artificial enamel caries lesions after infiltration with experimental light curing resins. - Caries Res., 42, 2008, 117-124.

9. Muelle r, J. et al. Inhibition of lesion progression by penetration of resins in vitro: Influence of the application procedure. - Oper. Dent., 31, 2006, 338-345, 12.

10. Neeraj Gugnani, Inder K. Pandit, Monika Gupta, Rohini Josan. Caries infiltration of noncavitated white spot lesions: A novel approach for immediate esthetic improvement. - Contemp. Clin. Dent., 3, 2012, (Suppl. 2), S199-S202. 
11. Paris, S. et H. Meyer-Lueckel. Inhibition of caries progression by resin infiltration in situ. - Caries Res., 44, 2010, № 1, 47-54.

12. Paris, S. et H. Meyer-Lueckel. Masking of labial enamel white spot lesions by resin infiltration: $A$ clinical report. - Quintessence Int., 40, 2009, 713-718.

13. Paris, S. et $H$. Meyer-Lueckel. The potential for resin infiltration technique in dental practice. Dent. Update., 39, 2012, № 9, 623-626, 628.

14. Rech m an $n$, P. et D. Charland. Performance of LIF device and visual examination for detection of occlusal caries in permanent molars. - J. Biomedical. Optics., 17, 2012, № 3, 1-15.

15. Sa i ir, S. et J. Shapira. Clinical solutions for developmental defects of enamel and dentin in children. - Pediatr. Dent., 29, 2007, 330-336.

16. Su bra m a n i a m, K., W. Siswomihardjo et S. Sunarintyas. The effect of different concentrations of Neem (Azadiractha indica) leaves extract on the inhibition of Streptococcus mutans (In vitro). - Maj. Ked. Gigi Dent. J., 38, 2005, 1769.

17. Terrer Ell o die, A. et al. A new concept in Restorative Dentistry:LIFEDT - Life-Induced Fluorescence Evaluator for Diagnosis and treatment. - J. Contemporary Practice, 11, 2010, № 1, 1-11-23.

18. Vasundhara Shivanna, B. Shivakumar. Novel treatment of white spot lesions: A report of two cases. - J. Conserv. Dent., 14, 2011, № 4, 423-426.

19. Vasundhara Shivanna, B Shivakumar. Novel treatment of white spot lesions: A report of two cases. - J. Conserv. Dent., 14, 2011, № 4, 423-426.

20. Young, D. A., V. K. Kutsch et J. Whitehouse. A clinician's guide to CAMBRA: A simple approach. - Compend. Contin. Educ. Dent., 30, 2009, 92-105.

\author{
Corresponding author: \\ Kalina Peycheva \\ Department Conservativ Dentistry \\ Faculty of Dental Medicine \\ Medical University \\ 1 Sv. G. Sofiiski St. \\ BG - 1431 Sofia \\ kelybg@yahoo.com
}

